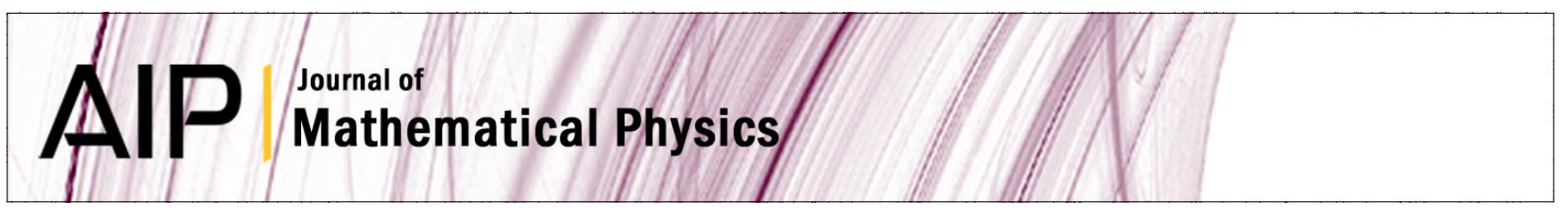

\title{
Dynamical systems embedded into Lie algebras
}

O. R. Campoamor-Stursberg, F. G. Gascon, and D. Peralta-Salas

Citation: J. Math. Phys. 42, 5741 (2001); doi: 10.1063/1.1412598

View online: http://dx.doi.org/10.1063/1.1412598

View Table of Contents: http://jmp.aip.org/resource/1/JMAPAQ/v42/i12

Published by the AIP Publishing LLC.

\section{Additional information on J. Math. Phys.}

Journal Homepage: http://jmp.aip.org/

Journal Information: http://jmp.aip.org/about/about_the_journal

Top downloads: http://jmp.aip.org/features/most_downloaded

Information for Authors: http://jmp.aip.org/authors

\section{ADVERTISEMENT}

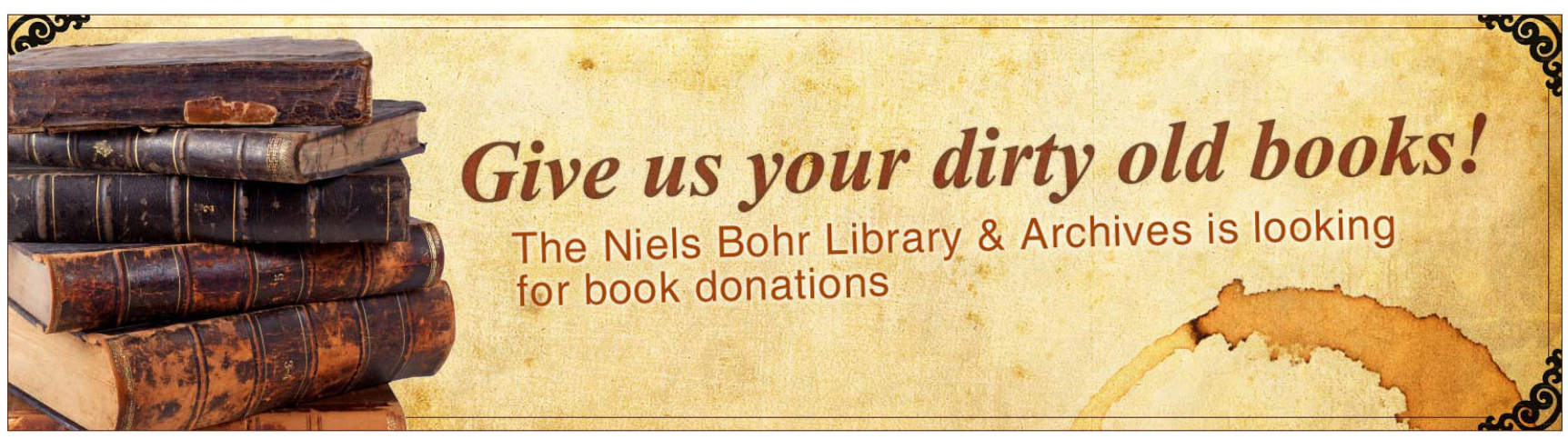




\title{
Dynamical systems embedded into Lie algebras
}

\author{
O. R. Campoamor-Stursberg \\ Facultad de CC. Matemáticas. Departemento de Geometría y Topología, Universidad \\ Complutense, Ciudad Universitaria, 28040, Madrid, Spain
}

F. G. Gascon and D. Peralta-Salas

Facultad de CC. Físicas, Departemento de Física Teórica II, Universidad Complutense, Ciudad Universitaria, 28040, Madrid, Spain

(Received 10 April 2000; accepted for publication 30 August 2001)

Analytical and geometrical information on certain dynamical systems $\mathbf{X}$ is obtained under the assumption that $\mathbf{X}$ is embedded into a certain real Lie algebra. (C) 2001 American Institute of Physics. [DOI: 10.1063/1.1412598]

\section{INTRODUCTION}

This article deals with the problem of extracting information of a three-dimensional dynamical system $\mathbf{X}$, when $\mathbf{X}$ is embedded into a Lie algebra of 3-D vectorfields.

This approach is interesting since up to now, as we explain later in this work, the only case considered has been that in which the generators of the Lie algebra are $\mathbf{X}$ and a certain number of symmetries or pseudosymmetries of $\mathbf{X}$. Such restriction is dropped in this article.

Let us explain this in more detail.

It is well known ${ }^{1}$ that when a vectorfield $\mathbf{X}$ (v.f. in what follows) admits a symmetry vector, that is, a v.f. $\mathbf{S}$ satisfying

$$
\mathcal{L}_{\mathbf{S}}(\mathbf{X})=0,
$$

$\mathcal{L}_{\mathbf{S}}$ standing for the Lie derivative along the streamlines of $\mathbf{S}$, useful consequences on the local and global structure of $\mathbf{X}$ can be obtained: existence of local and global first integrals, limit cycles of $\mathbf{X},{ }^{2}$ etc.

Remember that (1) implies that the flow of the v.f. $\mathbf{S}$ acts on the set of solutions of the differential equations

$$
\frac{d \mathbf{x}}{d t}=\mathbf{X}(\mathbf{x})
$$

In other words, the local flow of $\mathbf{S}$ transforms a solution of (2) into another solution of Eq. (2).

Sometimes the pair of v.f. (X,S) does not satisfy Eq. (1) but the equations

$$
\mathcal{L}_{\mathbf{S}}(\mathbf{X})=\lambda(\mathbf{x}) \mathbf{X}
$$

$\lambda(\mathbf{x})$ being a function. In this case $\mathbf{S}$ is called a pseudosymmetry of $\mathbf{X}$. The geometrical meaning of Eq. (3) is that the local flow of $\mathbf{S}$ conserves not the solutions of (2) but the trajectories on which these solutions lie (a trajectory of $\mathbf{X}$ is just an unparametrized solution of $\mathbf{X}$ ).

Interesting geometric information on the trajectories of $\mathbf{X}$ when (3) holds can be found in Ref. 2.

Motivated by Eqs. (1) and (3) we consider in this article that $\mathbf{X}$ (a $\mathbb{R}^{3}$ v.f. from now on) is one of the generators of a Lie algebra $A_{2,2}$ of dimension two or $A_{3,3}$ of dimension three. That is,

$$
\left[\mathbf{X}, \mathbf{S}_{1}\right]=a_{0} \mathbf{X}+a_{1} \mathbf{S}_{1}
$$




$$
\begin{gathered}
a_{0}, a_{1} \in \mathbb{R}, \\
\operatorname{rank}\left(\mathbf{X}, \mathbf{S}_{1}\right)=2 \text { for any } \mathbf{x} \in \mathbb{R}^{3},
\end{gathered}
$$

in the first case, and

$$
\begin{gathered}
{\left[\mathbf{X}, \mathbf{S}_{1}\right]=a_{0} \mathbf{X}+a_{1} \mathbf{S}_{1}+a_{2} \mathbf{S}_{2},} \\
{\left[\mathbf{X}, \mathbf{S}_{2}\right]=b_{0} \mathbf{X}+b_{1} \mathbf{S}_{1}+b_{2} \mathbf{S}_{2},} \\
{\left[\mathbf{S}_{1}, \mathbf{S}_{2}\right]=c_{0} \mathbf{X}+c_{1} \mathbf{S}_{1}+c_{2} \mathbf{S}_{2},} \\
a_{i}, b_{i}, c_{i} \in \mathbb{R}, \\
\operatorname{rank}\left(\mathbf{X}, \mathbf{S}_{1}, \mathbf{S}_{2}\right)=3 \text { for any } \mathbf{X} \in \mathbb{R}^{3},
\end{gathered}
$$

in the case of an algebra of type $A_{3,3}$.

Note that [,] stands for the Lie bracket of v.f. and $A_{i, j}(i \geqslant j)$ stands for a Lie algebra with $i$ generators (including $\mathbf{X})$ and $\operatorname{rank}\left(\mathbf{X}, \mathbf{S}_{1}, \ldots, \mathbf{S}_{i-1}\right)=j$.

We shall say that $\mathbf{X}$ belongs to a certain Lie algebra if $\mathbf{X}$ is one of its generators. For example, $\mathbf{X}$ belongs to the Lie algebras $A_{2,2}$ and $A_{3,3}$ defined by Eqs. (4) and (5).

Note that the case of pseudosymmetries corresponds to $a_{1}=0$ in Eq. (4) and $a_{1}=a_{2}=b_{1}$ $=b_{2}=0$ in Eq. (5).

We shall prove in what follows that when a dynamical system $\mathbf{X}$ belongs to a Lie algebra this information can be useful in order to get qualitative information on the orbits of $\mathbf{X}$.

This article is organized this way. Lie algebras of type $A_{2,2}$ are briefly considered in Sec. II, where their influence on $\mathbf{X}$ is studied. The structure constants of $A_{3,3}$ algebras are reduced to a finite number of canonical forms in Sec. III. The case of a v.f. $\mathbf{X}$ embedded into an $A_{3,3}$ Lie algebra is studied in Sec. IV. Illustrative examples are given in Sec. V, and some open problems are discussed in Sec. VI.

We end this section by motivating our study with some considerations of the significance and applicability of the idea of embedding a v.f. $\mathbf{X}$ into a Lie algebra.

We shall refer to the illustrative example of $A_{2,2}$ algebras [that is, algebras with two generators and rank equal 2: see Eq. (4)]. For these algebras Eq. (4) can be interpreted in two ways:

(i) as the structure equation of a Lie transformation (local) group $G$ acting on $\mathbb{R}^{3}$ of generators $\mathbf{X}$ and $\mathbf{S}$, or

(ii) as the equations defining an involutive distribution ${ }^{3,4}$ generated by $\mathbf{X}$ and $\mathbf{S}$.

The fact that $a_{0}$ and $a_{1}$ in Eq. (4) are real numbers instead of functions of $\mathbf{x}=\left(x_{1}, x_{2}, x_{3}\right)$ is a useful piece of information that should be taken into account.

Therefore the philosophy of this article is the following:

(i) get $\mathbf{X}$ (if you can, via computer packages, etc.) be embedded into the algebras $A_{r, 3}(r$ $\geqslant 3$ ) or $A_{r, 2}(r \geqslant 2)$ of some Lie transformation group $G$. We shall speak immediately about the difficulties of this process.

(ii) apply the techniques of this article in order to get information on some structures of $\mathbf{X}$, as first integrals, invariant sets, existence of partitions of $\mathbb{R}^{3}$ invariant under $\mathbf{X}$, integrability via quadratures, etc.

The most difficult point is, of course, the finding of the concrete embedding of $\mathbf{X}$. In fact it may even happen that (for structural reasons connected with the orbit structure of $\mathbf{X}$, strange or complicated limit behavior of the orbits when $t \rightarrow+\infty$ ) the embedding process will be a failure because it does not exist at all. For example, by topological reasons it is impossible to get an embedding of $\mathbf{X}$ into an algebra of type $A_{3,2}$ or $A_{2,2}$ if $\mathbf{X}$ is a dynamical system with an orbit which is an asymptotic "limit cycle" (orbit of type $S^{1}$ acting as limit set of neighboring orbits). Nevertheless, the dynamical system $\mathbf{X}$ could be embedded into an algebra of type $A_{3,3}$. 
However, we have not been able to find analytical conditions, geometric structures, etc. such that if $\mathbf{X}$ satisfies them, then $\mathbf{X}$ cannot be embedded into an algebra of type $A_{3,3}$. Upto today open problems are to decide

(i) whether or not a given v.f. $\mathbf{X}$ can be embedded into a finite dimensional Lie algebra, and (ii) whether or not a given v.f. $\mathbf{X}$ can be embedded into an algebra of type $A_{n, 3}(n \geqslant 3)$, where $n$ is a fixed natural number.

In general, the problem of studying the relation between the geometry of the orbits of $\mathbf{X}$ and the type of algebra into which $\mathbf{X}$ can or cannot be embedded seems to be a very difficult one.

In conclussion, this article could be of interest to people working in differential equations, dynamical systems, etc., and to all those normally handling symmetry techniques in differential equations since we offer here a certain generalization of them yielding, under some conditions, first integrals, invariant sets, integrability via quadratures, foliations of $\mathrm{R}^{3}$ invariant under $\mathbf{X}$, etc.

\section{II. $\mathrm{R}^{3}$ DYNAMICAL SYSTEMS EMBEDDED INTO A LIE ALGEBRA $\boldsymbol{A}_{2,2}$}

Let us now develop some consequences of the fact that our dynamical system $\mathbf{X}$ is embedded into a Lie algebra of type $A_{2,2}$, that is,

$$
\begin{gathered}
{\left[\mathbf{X}, \mathbf{S}_{1}\right]=a_{0} \mathbf{X}+a_{1} \mathbf{S}_{1},} \\
a_{0}, a_{1} \in \mathbb{R}, \\
\operatorname{rank}\left(\mathbf{X}, \mathbf{S}_{1}\right)=2 .
\end{gathered}
$$

We shall now obtain from Eq. (6) consequences of several kinds concerning the orbit structure of $\mathbf{X}$. Most of these results fail when the real constants $a_{0}$ and $a_{1}$ of (6) are substituted by real functions $a(\mathbf{x})$ and $b(\mathbf{x}), \mathbf{x} \in \mathbb{R}^{3}$. Therefore, most of these results cannot be obtained when $\mathbf{X}$ is embedded into a two-dimensional foliation instead of being embedded into a $A_{2,2}$ algebra.

From now on all the functions v.f.'s, and differential forms of this article are assumed to be analytic $\left(C^{w}\right)$. See Refs. 3-5 for the theory and applications of differential forms.

\section{A. First integrals of $X$}

We obtain now first integrals of $\mathbf{X}$ via the construction of exact one-forms. The reader can have a look at this method when $a_{0}=a_{1}=0$ in Ref. 3 .

Our assumptions are the following:

$\mathbf{X}$ belongs to a $A_{2,2}$ Lie algebra [see Eq. (6)] and

$$
\operatorname{Div} \mathbf{X}=-a_{1}, \operatorname{Div} \mathbf{S}_{1}=a_{0},
$$

$a_{0}$ and $a_{1}$ being the real numbers of Eq. (6) and Div $\mathbf{Y}$ standing for

$$
\begin{gathered}
\operatorname{Div} \mathbf{Y}=\frac{\partial Y_{1}}{\partial x_{1}}+\frac{\partial Y_{2}}{\partial x_{2}}+\frac{\partial Y_{3}}{\partial x_{3}}, \\
\mathbf{Y}=Y_{1} \partial_{1}+Y_{2} \partial_{2}+Y_{3} \partial_{3} .
\end{gathered}
$$

Div $\mathbf{Y}$ can be alternatively defined by $\mathcal{L}_{Y} \Omega_{3}=\operatorname{Div} \mathbf{Y} \cdot \Omega_{3}, \Omega_{3}$ being the standard volume form $d x_{1} \wedge d x_{2} \wedge d x_{3}$ of $\mathbb{R}^{3}$.

Under these hypotheses the one-form $w_{1}$ defined by

$$
w_{1}=i_{\mathbf{x}} i_{\mathbf{s}_{1}} \Omega_{3}
$$

is exact $\left(d w_{1}=0\right)$ and we can write 


$$
w_{1}=d I
$$

and since $i_{x} w_{1}=0$ we can write

$$
\mathcal{L}_{\mathbf{x}}(I)=0 .
$$

Therefore $I$ is a global first integral of $\mathbf{X}$.

Note that $I$ can never become a trivial constant, as this would imply $w_{1}=0$ (identically), getting a contradiction with the rank condition appearing in Eq. (6).

\section{B. Independent first integrals}

Let us now assume that $I_{1}, I_{2}$ are two independent first integrals of $\mathbf{S}_{\mathbf{1}}$; this situation often appears in physics ${ }^{6}$ as $\mathbf{S}_{\mathbf{1}}$ usually is a v.f. easier to handle than $\mathbf{X}$ (isometries of $\mathbb{R}^{3}$ considered as Euclidean space, linear or affine v.f. and so on). Under this assumption let us see that the integration of $\mathbf{X}$ can be simplified.

Under these conditions Eq. (6) implies

$$
-\mathcal{L}_{\mathbf{S}_{\mathbf{1}}} \mathcal{L}_{\mathbf{X}}\left(I_{i}\right)=a_{0} \mathcal{L}_{\mathbf{X}}\left(I_{i}\right), \quad i=1,2
$$

and when $a_{0}=0$ we get

$$
\mathcal{L}_{\mathbf{X}}\left(I_{i}\right)=\varphi_{i}\left(I_{1}, I_{2}\right)
$$

that is, $\mathbf{X}$ projects to the $R^{3}$ v.f.

$$
\mathbf{X}_{2}=\varphi_{1}\left(I_{1}, I_{2}\right) \partial_{I_{1}}+\varphi_{2}\left(I_{1}, I_{2}\right) \partial_{I_{2}},
$$

that is

$$
\begin{aligned}
& \frac{d I_{1}}{d t}=\varphi_{1}\left(I_{1}, I_{2}\right), \\
& \frac{d I_{2}}{d t}=\varphi_{2}\left(I_{1}, I_{2}\right) .
\end{aligned}
$$

Therefore, the integration of $\mathbf{X}$ has been simplified.

We now summarize the results of this section: We have seen that it is, in general, impossible to get geometric information on the trajectories of the $R^{3}$ v.f. $\mathbf{X}$ just by knowing that $\mathbf{X}$ belongs to a certain Lie algebra of v.f. More information concerning the v.f. of the Lie algebra is needed: see, for example, the requirements in (7).

A similar observation can be made in relation to the study of the pseudosymmetries of $\mathbf{X}$ [see Eq. (3)]. Namely, pseudosymmetries, per se, are insufficient in order to get first integrals and other geometric structures related to the trajectories of $\mathbf{X}$.

What is new in this section is the fact that we have shown the possibility of getting global geometric information on the trajectories of $\mathbf{X}$ when no pseudosymmetries are known but we have discovered that our dynamical system $\mathbf{X}$ is a generator of an $A_{2,2}$ algebra of vectorfields.

For brevity reasons we shall not study in the following sections algebras of type $A_{3,2}$, but just algebras of type $A_{3,3}$.

\section{CLASSIfICATION OF $\boldsymbol{A}_{3,3}$ ALGEBRAS}

A classification list of the $A_{3,3}$ algebras is given now. The proof shall not be given and will be sent on request. As we can see the classification contains 18 different types. Note that the non- 
written brackets between $\mathbf{X}, \mathbf{S}_{\mathbf{1}}$ and $\mathbf{S}_{\mathbf{2}}$ vanish and have been omitted. Nevertheless, all brackets have been written in the algebra of type number one (for esthetic reasons).

Any $A_{3,3}$ algebra can be obtained from those appearing in the list by means of linear combinations of type

$$
\begin{gathered}
\mathbf{X}^{*}=\alpha_{0} \mathbf{X}, \\
\mathbf{S}_{1}^{*}=\beta_{0} \mathbf{X}+\beta_{1} \mathbf{S}_{1}+\beta_{2} \mathbf{S}_{2}, \\
\mathbf{S}_{2}^{*}=\gamma_{0} \mathbf{X}+\gamma_{1} \mathbf{S}_{1}+\gamma_{2} \mathbf{S}_{2}, \\
\alpha_{0}, \beta_{0}, \gamma_{0} \in \mathbb{R} \quad \alpha_{0} \neq 0, \\
\beta_{1} \gamma_{2}-\gamma_{1} \beta_{2} \neq 0 .
\end{gathered}
$$

These linear combinations arise as the generator $\mathbf{X}$ (representing the dynamical system) must be isolated in all the algebraic manipulations; otherwise a generator $\mathbf{X}^{*}$ could be obtained mixing the dynamics of $\mathbf{X}$ with the dynamics of the v.f. $\mathbf{S}_{\mathbf{1}}$ and $\mathbf{S}_{\mathbf{2}}$. Therefore, the orbit structure of $\mathbf{X}$ would be unrelated to the orbit structure of $\mathbf{X}^{*}$.

The 18 types of $A_{3,3}$ algebras are

(1) $\left[\mathbf{X}, \mathbf{S}_{\mathbf{i}}\right]=0,\left[\mathbf{S}_{\mathbf{1}}, \mathbf{S}_{\mathbf{2}}\right]=0, i=1,2$;

(2) $\left[\mathbf{X}, \mathbf{S}_{\mathbf{1}}\right]=\mathbf{X}$;

(3) $\left[\mathbf{S}_{1}, \mathbf{S}_{2}\right]=\mathbf{X}$;

(4) $\left[\mathbf{X}, \mathbf{S}_{\mathbf{1}}\right]=\mathbf{S}_{\mathbf{1}}$;

(5) $\left[\mathbf{X}, \mathbf{S}_{2}\right]=\mathbf{S}_{1},\left[\mathbf{S}_{1}, \mathbf{S}_{2}\right]=\alpha \mathbf{S}_{1}, \alpha \in \mathbb{R}$;

(6) $\left[\mathbf{S}_{1}, \mathbf{S}_{2}\right]=\mathbf{S}_{1}$;

(7) $\left[\mathbf{X}, \mathbf{S}_{\mathbf{2}}\right]=\mathbf{X},\left[\mathbf{S}_{\mathbf{1}}, \mathbf{S}_{\mathbf{2}}\right]=\mathbf{X}+\alpha \mathbf{S}_{1}, \alpha \in \mathbb{R} \backslash\{0\}$;

(8) $\left[\mathbf{X}, \mathbf{S}_{\mathbf{2}}\right]=\mathbf{X}+\mathbf{S}_{\mathbf{1}},\left[\mathbf{S}_{\mathbf{1}}, \mathbf{S}_{\mathbf{2}}\right]=\mathbf{X}$;

(9) $\left[\mathbf{X}, \mathbf{S}_{\mathbf{2}}\right]=\mathbf{S}_{\mathbf{1}},\left[\mathbf{S}_{\mathbf{1}}, \mathbf{S}_{\mathbf{2}}\right]=-\mathbf{X}$;

(10) $\left[\mathbf{X}, \mathbf{S}_{\mathbf{2}}\right]=\mathbf{S}_{\mathbf{1}},\left[\mathbf{S}_{\mathbf{1}}, \mathbf{S}_{\mathbf{2}}\right]=\mathbf{X}$;

(11) $\left[\mathbf{X}, \mathbf{S}_{\mathbf{1}}\right]=\mathbf{S}_{\mathbf{1}},\left[\mathbf{X}, \mathbf{S}_{\mathbf{2}}\right]=\alpha \mathbf{S}_{\mathbf{2}}, \alpha \in \mathbb{R} \backslash\{0\}$;

(12) $\left[\mathbf{X}, \mathbf{S}_{\mathbf{1}}\right]=\mathbf{S}_{\mathbf{1}},\left[\mathbf{X}, \mathbf{S}_{\mathbf{2}}\right]=\mathbf{S}_{\mathbf{1}}+\mathbf{S}_{\mathbf{2}}$;

(13) $\left[\mathbf{X}, \mathbf{S}_{\mathbf{1}}\right]=\alpha \mathbf{S}_{\mathbf{1}}+\mathbf{S}_{\mathbf{2}},\left[\mathbf{X}, \mathbf{S}_{\mathbf{2}}\right]=-\mathbf{S}_{\mathbf{1}}+\alpha \mathbf{S}_{\mathbf{2}}, \alpha \in \mathbb{R} \backslash\{0\}$;

(14) $\left[\mathbf{X}, \mathbf{S}_{\mathbf{1}}\right]=\mathbf{X},\left[\mathbf{X}, \mathbf{S}_{\mathbf{2}}\right]=\mathbf{S}_{\mathbf{1}},\left[\mathbf{S}_{\mathbf{1}}, \mathbf{S}_{\mathbf{2}}\right]=\mathbf{X}+\mathbf{S}_{\mathbf{2}}$;

(15) $\left[\mathbf{X}, \mathbf{S}_{\mathbf{1}}\right]=\mathbf{S}_{\mathbf{2}},\left[\mathbf{X}, \mathbf{S}_{\mathbf{2}}\right]=-\mathbf{S}_{\mathbf{1}},\left[\mathbf{S}_{\mathbf{1}}, \mathbf{S}_{\mathbf{2}}\right]=\mathbf{X}$;

(16) $\left[\mathbf{X}, \mathbf{S}_{1}\right]=\mathbf{S}_{2},\left[\mathbf{X}, \mathbf{S}_{2}\right]=\mathbf{S}_{1},\left[\mathbf{S}_{1}, \mathbf{S}_{2}\right]=\mathbf{X}$;

(17) $\left[\mathbf{X}, \mathbf{S}_{1}\right]=-\mathbf{S}_{1}-\mathbf{S}_{2},\left[\mathbf{X}, \mathbf{S}_{\mathbf{2}}\right]=\mathbf{S}_{2},\left[\mathbf{S}_{\mathbf{1}}, \mathbf{S}_{\mathbf{2}}\right]=\mathbf{X}$; and

(18) $\left[\mathbf{X}, \mathbf{S}_{\mathbf{1}}\right]=-\mathbf{S}_{\mathbf{1}}+\mathbf{S}_{\mathbf{2}},\left[\mathbf{X}, \mathbf{S}_{\mathbf{2}}\right]=\mathbf{S}_{\mathbf{2}},\left[\mathbf{S}_{\mathbf{1}}, \mathbf{S}_{\mathbf{2}}\right]=\mathbf{X}$.

\section{INVARIANT SETS AND FIRST INTEGRALS WHEN THE DYNAMICAL SYSTEM IS EMBEDDED INTO AN $A_{3,3}$ ALGEBRA}

We now show that it is possible to get first integrals, invariant sets and foliations invariant under $\mathbf{X}$ when $\mathbf{X}$ belongs to an $A_{3,3}$ algebra. Reduction of $\mathbf{X}$ to a two-dimensional v.f. is also possible (see Sec. IV C).

\section{A. Global results}

We get in this paragraph global results on $\mathbf{X}$ assuming that

$$
\mathcal{L}_{\mathbf{X}} w_{i}=f(\mathbf{x}) w_{i},
$$

$w_{i}$ being a $\mathrm{C}^{\infty}$ differential form of degree $i(i=1,2,3)$.

Define the functions $\Delta_{i}$ via 


$$
\begin{gathered}
\Delta_{l}=i_{\mathbf{X}} i_{\mathbf{S}_{1}} i_{\mathbf{S}_{\mathbf{2}}} w_{3}, \\
\Delta_{2}=i_{\mathbf{X}} i_{\mathbf{S}_{j}} w_{2}, \quad j=1,2, \\
\Delta_{3}=i_{\mathbf{S}_{1}} i_{\mathbf{S}_{2}} w_{2}, \\
\Delta_{4}=i_{\mathbf{X}} w_{1}, \\
\Delta_{5}=i_{\mathbf{S}_{j}} w_{1}, \quad j=1,2 .
\end{gathered}
$$

We then get under standard manipulations ${ }^{4}$

$$
\mathcal{L}_{\mathbf{X}}\left(\Delta_{i}\right)=(f(\mathbf{x})+K) \Delta_{i}, \quad K \in \mathbb{R},
$$

where the real number $K$ depends on the constants $a_{i}, b_{i}, c_{i}(i=0,1,2)$ defining the $A_{3,3}$ algebra [see Eq. (5)].

Now, Eq. (23) implies the following.

(i) When the set $\left\{\Delta_{i}=0\right\}$ is a differential manifold $\left(\nabla\left(\Delta_{i}\right) \neq \mathbf{0}\right.$ for any $\left.P \in\left\{\Delta_{i}=0\right\}\right)$, then the set $\left\{\Delta_{i}=0\right\}$ is invariant under $\mathbf{X}$. See Example 1 in Sec. V.

(ii) When $f+K$ is a function of $\Delta_{i}$ (in particular when $f+K$ is a constant real number), then the sets $\left\{\Delta_{i}=\right.$ const $\}$ form a two-foliation invariant under $\mathbf{X}$.

(iii) When $f(\mathbf{x})$ is a trivial constant function and $f+K$ is equal to zero, then the function $\Delta_{i}$ is a global first integral of $\mathbf{X}$.

These results give useful information on the orbits of $\mathbf{X}$ and they have been obtained without problems in spite of the fact that $\mathbf{S}_{\mathbf{1}}$ and $\mathbf{S}_{\mathbf{2}}$ are, in general, not pseudosymmetries of $\mathbf{X}$.

See the examples on these results at the end of the article.

Note that the techniques of this section can be applied to any of the canonical algebras of the list in Sec. III.

\section{B. Subalgebras}

We now assume that our $A_{3,3}$ algebra contains two $A_{2,2}$ subalgebras satisfying

$$
\begin{gathered}
{\left[\mathbf{X}, \mathbf{S}_{\mathbf{1}}\right]=a \mathbf{X}+b \mathbf{S}_{\mathbf{1}},} \\
{\left[\mathbf{S}_{2}, \mathbf{X}\right]=a^{\prime} \mathbf{X}+b^{\prime} \mathbf{S}_{\mathbf{1}},} \\
{\left[\mathbf{S}_{2}, \mathbf{S}_{\mathbf{1}}\right]=a^{\prime \prime} \mathbf{X}+b^{\prime \prime} \mathbf{S}_{\mathbf{1}},} \\
a, a^{\prime}, a^{\prime \prime}, b, b^{\prime}, b^{\prime \prime} \in \mathbb{R},
\end{gathered}
$$

or

$$
\begin{aligned}
& {\left[\mathbf{S}_{1}^{*}, \mathbf{S}_{2}^{*}\right]=c \mathbf{S}_{1}^{*}+d \mathbf{S}_{2}^{*},} \\
& {\left[\mathbf{X}, \mathbf{S}_{1}^{*}\right]=c^{\prime} \mathbf{S}_{1}^{*}+d^{\prime} \mathbf{S}_{2}^{*},} \\
& {\left[\mathbf{X}, \mathbf{S}_{2}^{*}\right]=c^{\prime \prime} \mathbf{S}_{1}^{*}+d^{\prime \prime} \mathbf{S}_{2}^{*},} \\
& c, c^{\prime}, c^{\prime \prime}, d, d^{\prime}, d^{\prime \prime} \in \mathbb{R},
\end{aligned}
$$


or both [i.e., $A_{3,3}$ might contain a subalgebra satisfying Eq. (24) and another two-dimensional subalgebra satisfying Eq. (25)]. Note that $\left\{\mathbf{X}, \mathbf{S}_{\mathbf{1}}\right\}$ in the case of Eq. (24) and $\left\{\mathbf{S}_{\mathbf{1}}^{*}, \mathbf{S}_{\mathbf{2}}^{*}\right\}$ in the case of Eq. (25) are ideals of dimension two of $A_{3,3} \cdot{ }^{7}$

First of all, notice that we can apply the techniques of Sec. II to the pair $\left(\mathbf{X}, \mathbf{S}_{\mathbf{1}}\right)$ of Eq. (24).

Note that Eqs. (24) are fulfilled by the algebras 1, 2, 3, 4, 5, 6, 7, 8, 9, 10 and 13 and Eqs. (25) are satisfied by the algebras $1,4,5,6,11$, and 12 .

On the other hand, algebras 13-18 satisfy neither Eqs. (24) nor Eqs. (25). The reader will have no difficulty in checking all these points.

We give now the geometric meaning of Eqs. (24) and (25). Calling $\mathcal{F}_{2}$ and $\mathcal{F}_{2}^{*}$ the twofoliations associated with the pairs $\left(\mathbf{X}, \mathbf{S}_{1}\right)$ and $\left(\mathbf{S}_{1}^{*}, \mathbf{S}_{2}^{*}\right)$, Eqs. (24) and (25) can be rewritten in the form

$$
\mathcal{L}_{\mathbf{S}_{\mathbf{2}}}\left(\mathcal{F}_{2}\right) \subset \mathcal{F}_{2},
$$

and

$$
\mathcal{L}_{\mathbf{X}}\left(\mathcal{F}_{2}^{*}\right) \subset \mathcal{F}_{2}^{*} .
$$

Accordingly, $\mathcal{F}_{2}$ and $\mathcal{F}_{2}^{*}$ can be locally integrated via the well known formulas ${ }^{8}$

$$
\begin{gathered}
\Delta^{-1} \cdot\left(i_{\mathbf{X}} i_{\mathbf{S}_{1}} \Omega_{3}\right)=d I, \\
\Omega_{3}=d x_{1} \wedge d x_{2} \wedge d x_{3}, \\
\Delta=i_{\mathbf{X}} i_{\mathbf{S}_{1}} i_{\mathbf{S}_{2}} \Omega_{3},
\end{gathered}
$$

and

$$
\Delta^{-1} \cdot\left(i_{\mathbf{S}_{1}^{*}} i_{\mathbf{S}_{2}^{*}} \Omega_{3}\right)=d I^{*},
$$

$I$ and $I^{*}$ satisfying

$$
\mathcal{L}_{\mathbf{X}}(I)=0
$$

and

$$
\mathcal{L}_{\mathbf{X}}\left(I^{*}\right)=f\left(I^{*}\right)
$$

for a certain function $f$.

The function $I$ is, of course, a local integral of $\mathbf{X}$ and it globalizes to a $\mathbb{R}^{3}$ first integral of $\mathbf{X}$ when the function $\Delta$ of Eq. (28) never vanishes.

On the other hand, the geometrical meaning of Eq. (31) is that the local flow of $\mathbf{X}$ acts on the level sets of $I^{*}$. When the function $f$ of (31) never vanishes, $\mathbf{X}$ is free from closed trajectories. If $f\left(I_{0}^{*}\right)=0$, then closed trajectories of $\mathbf{X}$ might appear on the level set $I^{*}=I_{0}^{*}$.

Note that $I$ and $I^{*}$ are genuine functions, not reducing to constant functions, since in an $A_{3.3}$ algebra the ranks of the pairs $(\mathbf{X}, \mathbf{S})$ and $\left(\mathbf{S}_{\mathbf{1}}^{*}, \mathbf{S}_{\mathbf{2}}^{*}\right)$ cannot be lower than 2 .

\section{Results}

We now get several results on the orbits of the dynamical system $\mathbf{X}$ assuming that a pair of first integrals common to $\mathbf{S}_{\mathbf{1}}$ and $\mathbf{S}_{\mathbf{2}}$ are known. For brevity's sake, the case of only a first integral $I$ common to $\mathbf{S}_{\mathbf{1}}$ and $\mathbf{S}_{\mathbf{2}}$ shall not be studied.

See Ref. 6 for a similar use of a pair of first integrals of a symmetry of a $\mathbb{R}^{3}$ dynamical system related to the Bessel, Poisson-Boltzmann, Emden-Fowler and Fermi-Thomas equations. This 
approach can be justified since in most of the applications the v.f. $\mathbf{S}_{\mathbf{i}}$ are simple v.f.; often they are affine, or even linear v.f., and therefore the finding of their first integrals is, in general, not difficult.

Consider that

$$
\mathcal{L}_{\mathrm{S}_{\mathrm{i}}}\left(I_{j}\right)=0, \quad i, j=1,2
$$

that is $I_{1}, I_{2}$ are independent first integrals common to $\mathbf{S}_{1}, \mathbf{S}_{2}$. We then get via Eq. (5) $\left(c_{0} \neq 0\right)$

$$
\mathcal{L}_{\mathbf{X}}\left(I_{i}\right)=\varphi_{i}\left(I_{1}, I_{2}\right) \quad i=1,2 .
$$

Therefore $\mathbf{X}$ can be written in the form

$$
\mathbf{X}=\varphi_{1}(x, y) \partial_{1}+\varphi_{2}(x, y) \partial_{2} .
$$

Accordingly, $\mathbf{X}$ has been reduced to a $R^{2}$ v.f.

\section{EXAMPLES}

Examples 1: Consider the conformal v.f. ${ }^{4}$

$$
\mathbf{X}=\left(x_{1}^{2}-x_{2}^{2}-x_{3}^{2}\right) \partial_{1}+\left(2 x_{1} x_{2}\right) \partial_{2}+\left(2 x_{1} x_{3}\right) \partial_{3}
$$

and the v.f.

$$
\begin{gathered}
\mathbf{S}_{1}=x_{3} \partial_{2}-x_{2} \partial_{3}, \\
\mathbf{S}_{2}=x_{1} \partial_{1}+x_{2} \partial_{2}+x_{3} \partial_{3},
\end{gathered}
$$

with commutation relations

$$
\left[\mathbf{X}, \mathbf{S}_{1}\right]=\mathbf{0}, \quad\left[\mathbf{X}, \mathbf{S}_{2}\right]=-\mathbf{X}, \quad\left[\mathbf{S}_{1}, \mathbf{S}_{2}\right]=\mathbf{0} .
$$

By application of the results obtained in Secs. IV A and IV B we get

$$
\Delta_{1}=i_{\mathbf{X}} i_{\mathbf{S}_{\mathbf{1}}} i_{\mathbf{S}_{\mathbf{2}}}\left(d x_{1} \wedge d x_{2} \wedge d x_{3}\right)=\left(x_{2}^{2}+x_{3}^{2}\right)\left(-x_{1}^{2}-x_{2}^{2}-x_{3}^{2}\right) .
$$

On the other hand,

$$
\mathcal{L}_{\mathbf{X}}\left(\Delta_{1}\right)=6 x_{1} \cdot \Delta_{1} .
$$

Therefore, the set $\Delta_{1}=0$ is invariant under $\mathbf{X}$. Note that the set $\Delta_{1}=0$ is just the $x_{1}$-axis.

Let us now get a local first integral of $\mathbf{X}$ by application of the methods of Sec. IV B. In fact, computing $i_{\mathbf{X}} i_{\mathbf{S}_{1}}\left(d x_{1} \wedge d x_{2} \wedge d x_{3}\right) / \Delta_{1}$ we get the differential form

$$
\frac{w_{1}}{\Delta_{1}}=\frac{2 x_{1} d x_{1}}{-x_{1}^{2}-x_{2}^{2}-x_{3}^{2}}+\frac{\left(-x_{2} d x_{2}-x_{3} d x_{3}\right)\left(x_{1}^{2}-x_{2}^{2}-x_{3}^{2}\right)}{\left(x_{2}^{2}+x_{3}^{2}\right)\left(-x_{1}^{2}-x_{2}^{2}-x_{3}^{2}\right)},
$$

which is locally exact $\left(w_{1} / \Delta_{1}=d I\right)$. Upon integration we get the local first integral $I$ that can be reduced to

$$
I^{\prime}=\frac{x_{2}^{2}+x_{3}^{2}}{x_{1}^{2}+x_{2}^{2}+x_{3}^{2}} .
$$

Example 2: Consider now the family of v.f. 


$$
\mathbf{X}=F\left(x_{3}\right)\left(x_{1}^{2}+x_{2}^{2}\right)^{n} x_{1} \partial_{1}+F\left(x_{3}\right)\left(x_{1}^{2}+x_{2}^{2}\right)^{n} x_{2} \partial_{2}+G\left(x_{3}\right)\left(x_{1}^{2}+x_{2}^{2}\right)^{n} \partial_{3}, \quad n=1,2,3, \ldots,
$$

where $F$ and $G$ are analytic and $G$ vanishes on the set $Z(Z \subset R)$.

Let $\mathbf{S}_{\mathbf{i}}(i=1,2)$ be the v.f.

$$
\begin{aligned}
& \mathbf{S}_{\mathbf{1}}=x_{1} \partial_{1}+x_{2} \partial_{2}, \\
& \mathbf{S}_{\mathbf{2}}=x_{2} \partial_{1}-x_{1} \partial_{2} .
\end{aligned}
$$

The three v.f. $\mathbf{X}, \mathbf{S}_{\mathbf{1}}, \mathbf{S}_{\mathbf{2}}$ form a commutative algebra. By applying to them the techniques of Secs. IV $\mathrm{A}$ and IV $\mathrm{B}$ we get the invariant set

$$
\Delta_{1}=G\left(x_{3}\right)\left(x_{1}^{2}+x_{2}^{2}\right)^{n+1}=0
$$

that is, the invariant sets

$$
\begin{gathered}
x_{1}^{2}+x_{2}^{2}=0, \\
x_{3}=z, \quad z \in Z .
\end{gathered}
$$

On the other hand, we can also write

$$
\frac{w_{1}}{\Delta_{1}}=d I
$$

$w_{1}$ standing for the one-form

$$
w_{1}=i_{\mathbf{x}} i_{\mathbf{s}_{\mathbf{2}}}\left(d x_{1} \wedge d x_{2} \wedge d x_{3}\right)
$$

We get in this way

$$
I=\frac{1}{2} L\left(x_{1}^{2}+x_{2}^{2}\right)-\int \frac{F\left(x_{3}\right)}{G\left(x_{3}\right)} d x_{3},
$$

$L$ standing for Neperian logarithm, that is, a local first integral of $\mathbf{X}$.

Example 3: We now give an example related to Sec. II B.

Let $H_{i}\left(x_{1}, x_{2}, x_{3}\right)$ be homogeneous polynomials of degrees $d_{1}$ and $d_{2}$. Define $\mathbf{X}$ and $\mathbf{S}$ viahe equations

$$
\begin{gathered}
\mathbf{X}=\nabla H_{1} \wedge \nabla H_{2}+a_{0}\left(x_{1} \partial_{1}+x_{2} \partial_{2}+x_{3} \partial_{3}\right), \\
\mathbf{S}=\nabla H_{1} \wedge \nabla H_{2}, \\
a_{0} \in \mathbb{R}, \quad \nabla=\text { gradient operator. }
\end{gathered}
$$

The reader will check that

$$
[\mathbf{X}, \mathbf{S}]=b \mathbf{S}, \quad b \in \mathbb{R} .
$$

Therefore the pair $(\mathbf{X}, \mathbf{S})$ forms an $A_{2,2}$ algebra.

Since $H_{1}$ and $H_{2}$ are first integrals of $\mathbf{S}$, we get from (50)

$$
\begin{aligned}
& \mathcal{L}_{\mathbf{x}}\left(H_{1}\right)=\varphi_{1}\left(H_{1}, H_{2}\right), \\
& \mathcal{L}_{\mathbf{x}}\left(H_{2}\right)=\varphi_{2}\left(H_{1}, H_{2}\right),
\end{aligned}
$$


that is, $\mathbf{X}$ projects to the $R^{2}$ v.f.

$$
\varphi_{1} \partial_{H_{1}}+\varphi_{2} \partial_{H_{2}}
$$

Note that the v.f. $\mathbf{X}$ of (49) is not trivial, as it is not a homogeneous v.f.

Note also that any first integral $I\left(H_{1}, H_{2}\right)$ of the reduced differential equations (51) is a first integral of $\mathbf{X}$.

Example 4: The considerations of Example 3 can be extended to nonhomogeneous functions in this way.

Let $H_{1}$ and $H_{2}$ be nonhomogeneous polynomials that can be transformed into homogeneous ones via a transformation of type

$$
\begin{gathered}
x_{1} \rightarrow x_{1}^{a}, \\
x_{2} \rightarrow x_{2}^{b}, \\
x_{3} \rightarrow x_{3}^{c}, \\
a, b, c \in \mathbb{R}^{+} .
\end{gathered}
$$

For example, the pairs

$$
H_{1}=x_{2} x_{3}, \quad H_{2}=x_{1}^{2}+x_{2}^{2}+x_{3}
$$

and

$$
H_{1}=x_{2}^{2}+x_{3}^{2}, \quad H_{2}=x_{1}^{2}-x_{3}
$$

become homogeneous under the transformations

$$
x_{1} \rightarrow x_{1}, \quad x_{2} \rightarrow x_{2}, \quad x_{3} \rightarrow_{3}^{2}
$$

and

$$
x_{1} \rightarrow x_{1} \quad x_{2} \rightarrow{ }_{2}^{2}, \quad x_{3} \rightarrow x_{3}^{2} .
$$

Under these circumstances the v.f. defined by

$$
\begin{gathered}
\mathbf{X}=\nabla H_{1} \wedge \nabla H_{2}+a_{0}\left(x_{1} \partial_{1}+x_{2} \partial_{2}+x_{3} \partial_{3}\right), \\
\mathbf{S}=\nabla H_{1} \wedge \nabla H_{2},
\end{gathered}
$$

commutes as in Eq. (50). Therefore, the conclusions in Example 3 are valid for the v.f. of Eq. (54). For example, the Lorenz dynamical system ${ }^{9}$

$$
\mathbf{X}_{L}=\sigma\left(x_{2}-x_{1}\right) \partial_{1}+\left(-x_{1} x_{3}+r x_{1}-x_{2}\right) \partial_{2}+\left(x_{1} x_{2}-b x_{3}\right) \partial_{3}, \quad \sigma, r, b \in \mathbb{R},
$$

for the following particular values of the parameters,

$$
\sigma=\frac{1}{2}, \quad r=0, \quad b=1,
$$

forms an $A_{2,2}$ algebra, of the type discussed in this example, with the v.f.

$$
\mathbf{S}=\nabla\left(x_{2}^{2}+x_{3}^{2}\right) \wedge \nabla\left(x_{1}^{2}-x_{3}\right)
$$

as the reader can check. 
Example 5: We end this section with a list of second order differential equations appearing in Physics (see in Ref. 6) admitting a symmetry vector $\mathbf{S}$ to which the methods of this article can be applied (see Sec. II B).

(5.1) $x^{2} y_{, x x}+x y_{, x}+x^{2} y=0$ :

$$
\text { Associated } \mathbf{X}: \mathbf{X}=\frac{-x u-x^{2} y}{x^{2}} \partial_{u}+u \partial_{y}+\partial_{x} \quad u=y_{, x} .
$$

Symmetry vector: $\mathbf{S}=y \partial_{y}+u \partial_{u}$.

Commutation relation: $[\mathbf{X}, \mathbf{S}]=\mathbf{0}$.

First integrals of $\mathbf{S}: I_{1}=x, \quad I_{2}=u / y$.

(5.2) $y_{, x x}+y_{, x} / x=e^{y}$.

Associated $\mathbf{X}: \mathbf{X}=\left(e^{y}-\frac{u}{x}\right) \partial_{u}+u \partial_{y}+\partial_{x}, \quad u=y_{, x}$.

Symmetry vector: $\mathbf{X}=x \partial_{x}-2 \partial_{y}-u \partial_{u}$.

Commutation relation: $[\mathbf{X}, \mathbf{S}]=\mathbf{X}$.

First integrals of $\quad \mathbf{S}: I_{1}=x^{2} e^{y}, \quad I_{2}=x u$.

(5.3) $y_{, x x}+(2 / x) y_{, x}+y^{n}=0$.

Associated $\mathbf{X}: \mathbf{X}=\left(-y^{n}-\frac{2 u}{x}\right) \partial_{u}+u \partial_{y}+\partial_{x} \quad u=y_{, x}$.

Symmetry vector: $\mathbf{S}=x \partial_{x}+\frac{2 y}{1-n} \partial_{y}+\frac{1+n}{1-n} u \partial_{u}$.

Commutation relation: $[\mathbf{X}, \mathbf{S}]=\mathbf{X}$.

First integrals ofS: $I_{1}=x^{2} y^{n-1}, \quad I_{2}=x^{n+1} u^{n-1}$.

(5.4) $y_{, x x}=x^{-1 / 2} y^{3 / 2}$.

Associated $\mathbf{X}: \quad \mathbf{X}=\left(x^{-1 / 2} y^{3 / 2}\right) \partial_{u}+u \partial_{y}+\partial_{x}, \quad u=y_{, x}$.

Symmetry vector: $\mathbf{S}=x \partial_{x}-3 y \partial_{y}-4 u \partial_{u}$.

Commutation relation: $[\mathbf{X}, \mathbf{S}]=\mathbf{X}$.

First integrals of $\mathbf{S}: I_{1}=x^{3} y, \quad I_{2}=x^{4} u$.

\section{FINAL REMARKS}

We have seen that when a $\mathbb{R}^{3}$ dynamical system $\mathbf{X}$ lies inside an $A_{2,2}, A_{3,2}$ or $A_{3,3}$ algebra useful information on its trajectories can be obtained from this piece of information.

What happens when $\mathbf{X}$ can be embedded into a Lie algebra $A_{n, 3}$ when $n>3$ ? Note that now the canonical forms of Sec. III are harder to obtain. On the other hand, $A_{n, 3}$ might contain ideals I containing $\mathbf{X}$ of lower dimension $n^{\prime}$, reducing the problem to an algebra $A_{n^{\prime}, 3}$ of lower dimension. If no ideal of this type can be found, we can always apply the techniques of Sec. IV A. 
Considering only contractions of $\mathbf{X}$ and $\mathbf{S}_{\mathbf{i}}$ with differential forms of type $w_{3}$, we can get in this way a whole set of functions $\Delta_{i j}$ :

$$
\Delta_{i j}=i_{X} i_{S_{i}} i_{S_{j}} w_{3}, \quad i, j=1, \ldots, n-1,
$$

leading to the sets

$$
\Delta_{i j}\left(x_{1}, x_{2}, x_{3}\right)=0
$$

that are invariant under $\mathbf{X}$ [at least near the points $P$ on which (58) defines a differential manifold, that is $\left.\nabla\left(\Delta_{i j}\right)(P) \neq 0\right]$.

Therefore, when $n$ is high we can get, via Eq. (58), a collection of more and more sets invariant under $\mathbf{X}$.

An open problem is to study if the number $N$ of invariant sets in (58) is bounded or not when $n$ increases and whether or not these invariant sets accumulate (when $N$ is unbounded). Does the topology of the trajectories of $\mathbf{X}$ "feel" that $\mathbf{X}$ is included in an $A_{n, 3}$ algebra (without proper ideals) when $n$ is large?

Another open problem meriting a separate study is this one: Assume that $\mathbf{X}$ is included among the generators of an $A_{\infty, 3}$ algebra where $A_{\infty, 3}$ is an infinite Lie algebra, free from finite or infinite proper ideals containing $\mathbf{X}$. Let us call them simple $\infty$-algebras.

Equation (57) can now be written in the form

$$
\Delta_{i j}=i_{X} i_{S_{i}} i_{S_{j}} w_{3},
$$

and, therefore, invariant sets of $\mathbf{X}$ can be obtained this way.

The question arises again of classifying topologically the v.f. $\mathbf{X}$ that can be included in a simple $A_{\infty, 3}$ algebra.

A final question is this one: can a dynamical system $\mathbf{X}$ embedded into a Lie algebra $A_{n, 2}, A_{n, 3}$ or $A_{\infty, 2}, A_{\infty, 3}$ possess a strange attractor? ${ }^{9}$

\section{ACKNOWLEDGMENT}

The authors thank one of the referees of the article for the his/her useful comments.

${ }^{1}$ P. Olver, Applications of Lie Groups to Differential Equations (Springer, New York, 1986).

${ }^{2}$ G. Unal, Phys. Lett. A 260, 352 (1999); G. Jones, Nuovo Cimento Soc. Ital. Fis., B 112B, 1053 (1997); F. G. Gascon, Nuovo Cimento Soc. Ital. Fis., B 29, 73 (1980); M. Lutzky, J. Phys. A 12, 973 (1979).

${ }^{3}$ V. Arnold and A. Avez, Problemes Ergodiques de la Mecanique Classique (Gauthier-Villars, Paris, 1967); V. Arnold, Methodes Mathematiques de la Mecanique Classique (MIR, Moscow, 1976).

${ }^{4}$ Y. Choquet-Bruhat, C. DeWitt-Morette, and M. Dillard-Bleick, Analysis, Manifolds and Physics (North Holland, Amsterdam, 1978); B. Harrison and F. Estabrook, J. Math. Phys. 12, 653 (1971).

${ }^{5}$ R. Abraham, Foundations of Mechanics (Benjamin, New York, 1967).

${ }^{6}$ T. Sen and M. Tabor, Physica D 44, 313 (1990); L. Dresner, J. Math. Phys. 12, 1339 (1971).

${ }^{7}$ N. Jacobson, Lie Algebras (Dover, New York, 1962); R. Gilmore, Lie Groups, Lie Algebras and Some of Their Applications (Wiley, New York, 1974).

${ }^{8}$ L. Eisenhart, Continuous Groups of Transformations (Princeton University Press, Princeton, NJ, 1933); S. Lie, Math. Ann. 25, 71 (1888); S. Lie and G. Scheffers, Vorlesungen uber differentialgleichungen mit bekanten infinitesimalen transformationen (Teubner, Leipzig, 1891).

${ }^{9}$ C. Sparrow, The Lorenz Equations: Bifurcation, Chaos and Strange Attractors (Springer, New York, 1982); E. Lorenz, Atmos. Sci. 20, 130 (1963); B. Hasard, J. Zhang, S. Hastings, and W. Troy, Appl. Math. Lett. 7, 79 (1994). 